\title{
Critically phase-matched Ti:sapphire-laser-pumped deep-infrared femtosecond optical parametric oscillator based on $\mathrm{CdSiP}_{2}$
}

\author{
Callum F. O'Donnell, ${ }^{1,2, *}$ S. Chaitanya Kumar, ${ }^{1,2}$ K. T. ZaWilski ${ }^{3}$, \\ P. G. SCHUNEMANN ${ }^{3}$ AND M. EBRAHIM-ZADEH ${ }^{1,2,4}$ \\ ${ }^{1}$ Radiantis, Polígon Camí Ral, 08850 Gavà, Barcelona, Spain \\ ${ }^{2}$ ICFO-Institut de Ciencies Fotoniques, The Barcelona Institute of Science and Technology, 08860 Castelldefels, Barcelona, Spain \\ ${ }^{3}$ BAE Systems, Incorporated, MER15-1813, P.O. Box 868, Nashua, New Hampshire 03061-0868, USA \\ ${ }^{4}$ ICREA, Passeig Lluis Companys 23, 08010 Barcelona, Spain \\ *Corresponding author: callum.odonnell@radiantis.com
}

Received XX Month XXXX; revised XX Month, XXXX; accepted XX Month XXXX; posted XX Month XXXX (Doc. ID XXXXX); published XX Month XXXX

\begin{abstract}
We report a high-repetition-rate femtosecond optical parametric oscillator (OPO) for the deep-infrared based on type-I critical phase-matching in $\mathrm{CdSiP}_{2}$ (CSP), pumped directly by a Ti:sapphire laser. Using angletuning in the CSP crystal, the OPO can be continuously tuned across 7306-8329 $\mathrm{nm}\left(1201-1369 \mathrm{~cm}^{-1}\right)$ in the deep-infrared. It delivers up to $18 \mathrm{~mW}$ of idler average power at $7306 \mathrm{~nm}$ and $>7 \mathrm{~mW}$ beyond $8000 \mathrm{~nm}$ at 80.5 $\mathrm{MHz}$ repetition rate, with the spectra exhibiting bandwidths of $>150 \mathrm{~nm}$ across the tuning range. Moreover, the signal is tunable across 1128-1150 nm in the near-infrared, providing up to $35 \mathrm{~mW}$ of average power in $\sim 266$ fs pulses at $1150 \mathrm{~nm}$. Both beams exhibit single-peak Gaussian distribution in TEMoo spatial profile. With an equivalent spectral brightness of $\sim 5.6 \times 10^{20}$ photons $\mathrm{s}^{-1} \mathrm{~mm}^{-2} \mathrm{sr}^{-1} 0.1 \% \mathrm{BW}^{-1}$, this OPO represents a viable alternative to synchrotron and supercontinuum sources for deep-infrared applications in spectroscopy, metrology and medical diagnostics. (C) 2017 Optical Society of America
\end{abstract}

OCIS codes: (190.4360) Nonlinear optics, devices; (190.7110) Ultrafast nonlinear optics; (190.4400) Nonlinear optics, materials; (190.4970) Parametric oscillators and amplifiers.

http://dx.doi.org/10.1364/OL.99.099999

The spectral region of 7-8.5 $\mu \mathrm{m}$ in the deep-infrared (deep-IR) is highly coveted for a variety of applications across many scientific and technological disciplines, including vibrational spectroscopy, frequency comb generation, and cancer diagnostics [1-3]. In order to enable rapid data acquisition with a high signal-to-noise ratio and low risk of laser-induced damage, such applications require high-average-power coherent sources at high repetition rate, with broad wavelength flexibility in ultrashort pulses [4]. With the scarcity of conventional ultrafast laser sources at wavelengths beyond $\sim 3 \mu \mathrm{m}$, nonlinear frequency conversion techniques offer the most viable approach to the generation of tunable ultrashort pulses in the mid-IR to deep-IR. In the high-repetition-rate picosecond and femtosecond regime, synchronously-pumped optical parametric oscillators (OPOs) pumped by near-IR ultrafast lasers such as the Kerr-lens-mode-locked (KLM) Ti:sapphire and $\mathrm{Yb}$-fiber lasers have emerged as the most effective solution, providing tunable coherent light of excellent spatial beam quality and high spectral brilliance. The potential of such systems has been demonstrated in frequency metrology and imaging of biological samples [5, 3].

Accessing the mid-IR and deep-IR wavelength regions beyond $4.5 \mu \mathrm{m}$ has proven challenging due to the onset of multiphonon absorption in oxide-based nonlinear materials, and a lack of readily available non-oxide-based crystals of high optical quality with transparency at the required pump, signal and idler wavelengths. The search for alternative materials for mid-IR to deep-IR generation led to the development of the new nonlinear crystal, $\mathrm{CdSiP}_{2}$ (CSP) [6], a chalcopyrite with a transparency range from $\sim 1$ to $\sim 6.5 \mu \mathrm{m}$, and an effective nonlinearity 4 times that of MgO:PPLN ( $d_{\text {eff }} \sim 84.5 \mathrm{pm} / \mathrm{V}$ ). Its high thermal conductivity (13.6 $\mathrm{W} / \mathrm{m} \cdot \mathrm{K}$ ) and bulk damage threshold (41 $\mathrm{MW} / \mathrm{cm}^{2}$ at $1064 \mathrm{~nm}$ ), together with the improvements in optical quality, have made CSP an increasingly attractive candidate for nonlinear frequency conversion into the mid-IR to deep-IR. Importantly, the bandgap of CSP is estimated to be $2.2-2.45 \mathrm{eV}$, implying that two-photon absorption is not significant until the pump wavelength is below $\sim 1 \mu \mathrm{m}$. As such, CSP can be pumped in the vicinity of $1 \mu \mathrm{m}$ to generate deep-IR radiation across nearly its entire transmission window by employing type-I $(e \rightarrow o o)$ noncritical phase-matching 
(NCPM) [7]. As we demonstrate in this work, as a negative uniaxial crystal with a birefringence of $n_{e}-n_{o}=-0.051$ at $7 \mu \mathrm{m}, \mathrm{CSP}$ also possesses the ability to generate broadly tunable deep-IR radiation by simple crystal rotation under type-I $(e \rightarrow o o)$ critical phasematching. To date, femtosecond OPOs based on CSP have been demonstrated only at a fixed angle $\left(\theta=90^{\circ}\right)$ under NCPM, with spectral tuning achieved via cavity delay or pump tuning. The first CSP-based femtosecond OPO generated a fixed idler wavelength of $6.2 \mu \mathrm{m}$ and was pumped at $1.053 \mu \mathrm{m}$ by a $100 \mathrm{MHz} \mathrm{Yb}: \mathrm{KYW}$ master laser followed by a Yb-doped fiber amplifier [8]. This was followed by an OPO pumped with a femtosecond Yb:KGW solidstate laser at $1.029 \mu \mathrm{m}$, providing deep-IR average powers of up to $110 \mathrm{~mW}$ at $7 \mu \mathrm{m}$ [9]. Later, a Ti:sapphire-pumped femtosecond Mg0:PPLN OPO was used to pump CSP, in both internal [10] and external [11] cascaded configurations at $\sim 80 \mathrm{MHz}$, producing $\mu$ Watt-level average power across 5.9-8.1 $\mu \mathrm{m}$ and up to $32 \mathrm{~mW}$ across 6.318-7.0 $\mu \mathrm{m}$, respectively. Furthermore, an alternative route to the deep-IR, using an OPO based on orientation-patterned $\mathrm{GaP}$ (OP-GaP), was reported to produce up to $55 \mathrm{~mW}$ at several discrete wavelengths across 5-12 $\mu \mathrm{m}$ [12].

In this work, we advance the state of the art in femtosecond OPOs by demonstrating a deep-IR OPO based on CSP pumped directly by a Kerr-lens-mode-locked (KLM) Ti:sapphire laser at $<1$ $\mu \mathrm{m}$, with no intermediate frequency conversion stage. By exploiting angle tuning under type-I $(e \rightarrow 00)$ critical phasematching in CSP, we have achieved spectral coverage across 7306$8329 \mathrm{~nm}$ in the deep-IR idler, generating up to $18 \mathrm{~mW}$ of average power at $7306 \mathrm{~nm}$ with $>7 \mathrm{~mW}$ at $8227 \mathrm{~nm}$. This wavelength range covers key regions of the deep-IR spectrum, including the amide III band, important for human surgery. To our knowledge, these results represent the longest wavelengths with multimilliwatt average powers generated from a Ti:sapphire-pumped femtosecond OPO based on any nonlinear crystal, and the highest practical power levels generated beyond $7 \mu \mathrm{m}$ using CSP.

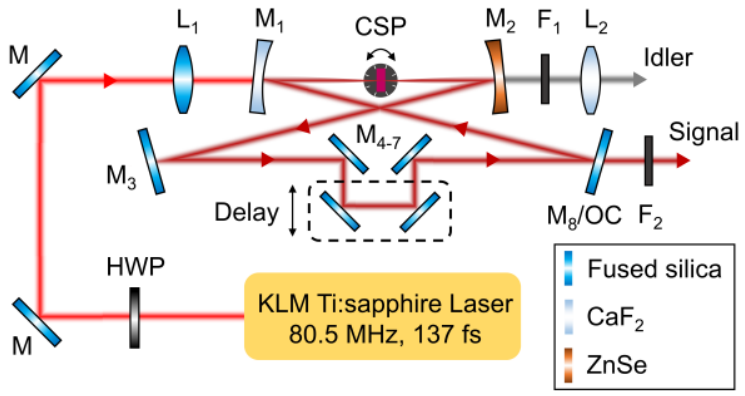

Fig. 1. Schematic of the experimental setup for the Ti:sapphirepumped CSP femtosecond OPO. HWP: Half wave plate, M: Mirrors, OC: Output coupler, L: Lenses, F: Filters.

The setup for the CSP-based femtosecond OPO is depicted in Fig. 1 . The OPO is synchronously pumped by $\Delta \tau \sim 137$ fs input pulses from a KLM Ti:sapphire laser (Spectra-Physics, Mai Tai HP) at a repetition rate of $80.5 \mathrm{MHz}$. For this experiment, we operated the laser at an optimally selected fixed wavelength of $993 \mathrm{~nm}$, where the measured full-width half-maximum (FWHM) spectral bandwidth was $\Delta \lambda \sim 10.5 \mathrm{~nm}$, corresponding to a time-bandwidth product of $\Delta v \Delta \tau \sim 0.44$, close to the transform limit for $\operatorname{sech}^{2}$ pulses.
After transmission through $\mathrm{L}_{1}$ and $\mathrm{M}_{1}$, the average pump power incident on the crystal is $\sim 690 \mathrm{~mW}$. The CSP sample is 1-mm-long with a $4 \mathrm{~mm} \times 5 \mathrm{~mm}$ aperture, and cut at $\theta=90^{\circ}\left(\phi=45^{\circ}\right)$ for type-I $(e \rightarrow 0 o)$ NCPM at normal incidence. The crystal is maintained at room temperature and mounted on a precision rotation stage, with both end faces antireflection (AR)-coated for high transmission at the pump ( $T>99 \%$ over $950-1080 \mathrm{~nm})$, signal ( $T>99 \%$ across $1100-1200 \mathrm{~nm}$ ), and idler (T>93\% across 7000 $8500 \mathrm{~nm}$ ). The pump polarization is controlled using a half-wave plate $(\lambda / 2)$ and the beam is focused to a waist radius of $w_{0} \sim 28 \mu \mathrm{m}$ at the center of the crystal using a lens ( $\left.\mathrm{L}_{1}\right)$ of focal length, $f=100$ $\mathrm{mm}$. The OPO cavity comprises two plano-concave mirrors $\left(\mathrm{M}_{1-2}\right)$ with radius of curvature, $r=100 \mathrm{~mm}$, and six plane mirrors $\left(\mathrm{M}_{3-8}\right)$. $\mathrm{M}_{1}$ transmits the pump (T 83\% at $993 \mathrm{~nm}$ ) and is highly reflecting (HR) for the signal ( $R>99 \%$ across $1100-1300 \mathrm{~nm}) . \mathrm{M}_{2}$ is a 3-mmthick ZnSe substrate, AR-coated for the deep-IR idler (T>94\% across $6500-9000 \mathrm{~nm})$, and HR for the signal $(R>97 \%$ across $1100-1300 \mathrm{~nm}) . \mathrm{M}_{8}$ is a $5 \%$ signal output coupler (OC) $(R=95 \%$ across 1100-1300 nm). All other mirrors ( $\left.M_{3-7}\right)$ are fused silica substrates, HR for the signal ( $R>99.8 \%$ across $1100-1300 \mathrm{~nm})$, and negatively chirped for dispersion compensation (GDD -100 $-400 \mathrm{fs}^{2}$ across $1100-1160 \mathrm{~nm}$ ). The OPO is thus singly-resonant for the near-IR signal wave, with the undepleted pump exiting the cavity through $\mathrm{M}_{8}$, while the single-pass deep-IR idler is transmitted through $\mathrm{M}_{2}$. Finally, after appropriate filtering, the idler is collimated using a $\mathrm{CaF}_{2}$ lens with good deep-IR transmission ( $T>60 \%$ over $6000-8500 \mathrm{~nm}$ ).

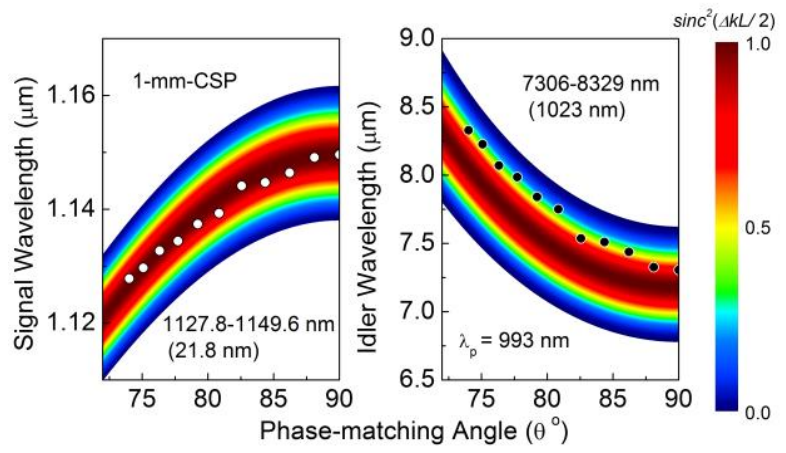

Fig. 2. Idler and signal tuning as a function of the internal phasematching angle in the CSP crystal, for a pump wavelength of $993 \mathrm{~nm}$, superimposed on the parametric gain map.

The OPO signal pulses were synchronized with the pump pulses by means of a delay line, consisting of mirrors M5 and M6 mounted on a precision translation stage, and oscillation was observed in the form of parasitic yellow light generated by nonphase-matched second harmonic generation of the signal. The output-coupled signal power was optimized by fine-tuning the pump wavelength, $\lambda_{\mathrm{p}}$, with the resultant selected value of $993 \mathrm{~nm}$ arising from a trade-off between higher pump powers and drop in crystal transmission as $\lambda_{\mathrm{p}}$ was decreased. Wavelength tuning of the OPO was realized by rotating the CSP crystal away from the NCPM condition, reducing the phase-matching angle from $\theta=90^{\circ}$ to $74^{\circ}$. At a phase-matching angle of $\theta=74^{\circ}$, the high refractive index of CSP $(n \sim 3.14)$ necessitated an external crystal orientation of $30^{\circ}$ to the pump beam. For each phase-matching angle, the signal spectrum at perfect synchronization was recorded, and the 
corresponding deep-IR idler was calculated from the central wavelength using energy conservation. The results are shown in Fig. 2, where it can be seen that the near-IR signal wavelength could be tuned across $1127.8-1149.6 \mathrm{~nm}$, with the idler covering a spectral range of 7306-8329 $\mathrm{nm}$ in the deep-IR. The measured values are superimposed on the parametric gain map calculated using the Sellmeier equation in [6].

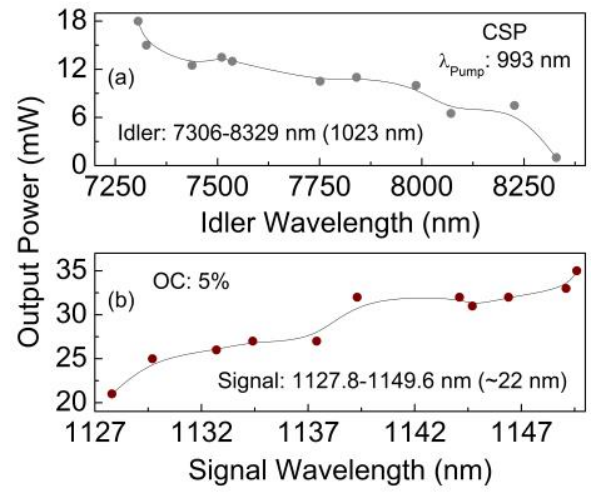

Fig. 3. (a) Deep-IR idler, and (b) near-IR signal average power across the tuning range of the CSP femtosecond OPO, using a 5\% OC. The solid line is a guide to the eye.

The signal and idler output powers across the tuning range of the CSP femtosecond OPO, for a fixed input pump power of 690 $\mathrm{mW}$, are displayed in Fig. 3. We extracted $>10 \mathrm{~mW}$ of deep-IR idler average power across $>60 \%$ of the tuning range, with a maximum of $18 \mathrm{~mW}$ at $7306 \mathrm{~nm}$ and $>6 \mathrm{~mW}$ available up to $8227 \mathrm{~nm}$. This is equivalent to a peak quantum conversion efficiency of $19.1 \%$. The drop in the idler power towards longer wavelengths is attributed to the increasing absorption of CSP in the deep-IR, the lowering of signal wavelength resulting in increased loss at the shortwavelength absorption edge of the crystal, as well as the rise in Fresnel reflection loss from the crystal facets at phase-matching angles far away from normal incidence. In addition to the deep-IR idler beam, we were able to extract up to $35 \mathrm{~mW}$ of output power in the near-IR signal at $1150 \mathrm{~nm}$ using the $5 \%$ OC, with $>25 \mathrm{~mW}$ over $>90 \%$ of the tuning range. It is to be noted that the data for the signal and idler power is not corrected for the coating or absorption losses in the crystal, filters, $\mathrm{F}_{1-2}$, and lens, $\mathrm{L}_{2}$, which can be more than $\sim 45 \%$. Power scaling measurements performed at signal and idler wavelengths of $1149 \mathrm{~nm}$ and $7314 \mathrm{~nm}$, respectively, are shown in Fig. 4. As shown in the inset, at the maximum input power, the pump depletion is estimated to be $\sim 48 \%$. Here, the input pump power is corrected for transmission loss through $\mathrm{M}_{1}$ (T 83\%). From the plot, evidence of saturation is observed in the signal, indicating that the output coupling of $5 \%$ is not sufficient for optimal signal extraction. The detailed spectral characteristics of the signal pulses extracted from the OPO, recorded using a spectrum analyzer with a resolution of $0.7 \mathrm{~nm}$, and are shown in Fig. 5, indicating FWHM bandwidths of $\sim 12-14$ $\mathrm{nm}$. The asymmetric spectrum with a long-wavelength tail is characteristic of self-phase-modulation arising from the Kerr nonlinearity in CSP, combined with the broad parametric gain bandwidth shown in Fig. 2.

The deep-IR idler spectrum, measured using a HeNe-calibrated wavelength meter with a resolution of $\sim 7 \mathrm{~cm}^{-1}$, has a typical
FWHM spectral bandwidth of $\sim 152 \mathrm{~nm}\left(\sim 28 \mathrm{~cm}^{-1}\right)$, centered at $7387 \mathrm{~nm}$, as presented in Fig. 6. The corresponding signal spectrum, and the TEM $\mathrm{T}_{00}$ idler beam profile, are shown in the inset. The strong modulations on the idler spectra are associated with water absorption lines in the $7250-7500 \mathrm{~nm}\left(1330-1380 \mathrm{~cm}^{-1}\right)$ wavelength range.

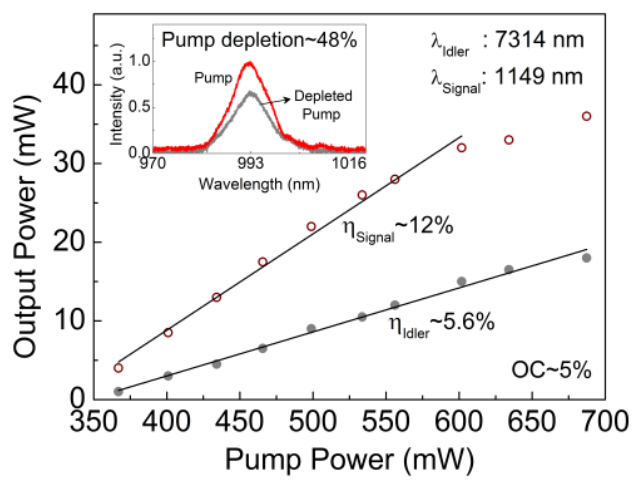

Fig. 4. Power scaling of the signal and idler extracted from the CSP femtosecond OPO. Inset: Input pump spectrum and depleted pump spectrum while the $\mathrm{OPO}$ is operating at maximum power.

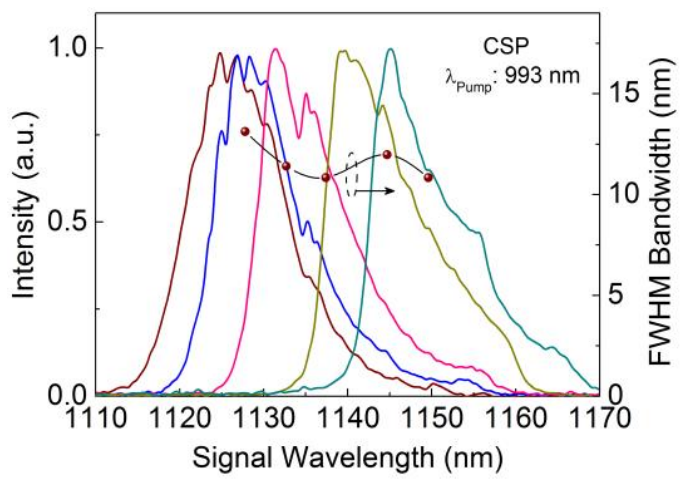

Fig. 5. Signal spectra and corresponding FWHM bandwidths across the tuning range of the critically phase-matched CSP femtosecond OPO.

To compare the brilliance of CSP OPO idler output to other deep-IR sources, the spectral brightness was calculated using the method in [12]. At a central wavelength of $7387 \mathrm{~nm}$, where a total average power of $17 \mathrm{~mW}$ was measured, from Fig. 7 it can be determined that $0.821 \mathrm{~mW}$ of optical power is contained within $\pm 0.1 \%$ bandwidth of the central frequency. This is equivalent to $\sim 3.05 \times 10^{16}$ photons $\mathrm{s}^{-1} 0.1 \% \mathrm{BW}^{-1}$. Using ABCD matrix analysis, the beam waist of the signal cavity is calculated to be $w_{0 \mathrm{~s}} \sim 23.4 \mu \mathrm{m}$, implying that the idler is generated with a waist radius of $w_{0 \mathrm{i}} \sim 18.0$ $\mu \mathrm{m}$ in the crystal $\left(1 / w_{0 i}^{2}=1 / w_{0 p^{2}}{ }^{2}+1 / w_{0 s}^{2}\right)$. Therefore, the idler beam solid angle is 0.0538 steradians, resulting in a spectral brightness of $\sim 5.60 \times 10^{20}$ photons s $\mathrm{mm}^{-1} \mathrm{sr}^{-1} 0.1 \% \mathrm{BW}^{-1}$ at 7387 $\mathrm{nm}$. This value of spectral brightness is $\sim 5$ orders of magnitude greater than a conventional FTIR Globar, and 2 orders of magnitude higher than a typical synchrotron facility [13]. Furthermore, it is comparable to the record brightness of $\sim 8 \times 10^{20}$ photons $\mathrm{s}^{-1} \mathrm{~mm}^{-2} \mathrm{sr}^{-1} 0.1 \% \mathrm{BW}^{-1}$ from a mid-IR 
supercontinuum source, with $\sim 20 \mathrm{~mW}$ of white light across 2-7.5 $\mu \mathrm{m}$ [14]. However, in terms of $0.1 \% \mathrm{BW}^{-1}$ average power at 7387 $\mathrm{nm}$, the CSP femtosecond OPO is $\sim 15$ times more powerful.

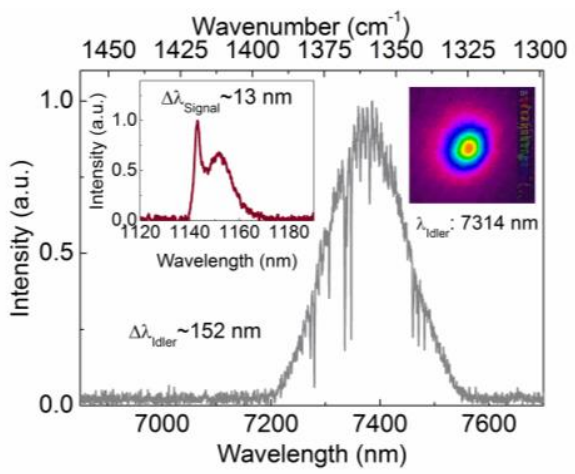

Fig. 6. Deep-IR idler spectrum centered on $7387 \mathrm{~nm}$ with a FWHM bandwidth of $152 \mathrm{~nm}$. Insets: Corresponding signal spectrum centered at $\sim 1150 \mathrm{~nm}$ with FWHM bandwidth of $13 \mathrm{~nm}$, TEM 00 beam profile at $7314 \mathrm{~nm}$

Finally, we performed temporal characterization of the CSP femtosecond OPO output using intensity autocorrelation, in order to estimate the signal pulse duration. A typical autocorrelation trace of the signal, recorded at $\sim 1145 \mathrm{~nm}$, is depicted in Fig. 7, with the corresponding spectrum presented in the inset. Assuming a $\operatorname{sech}^{2}$ pulse shape, this measurement yields a duration of $\sim 259 \mathrm{fs}$, implying a strongly chirped pulse. Together with the spectral measurements in Fig. 5, this provides strong evidence of net positive cavity dispersion. Using an estimated total cavity dispersion of $\sim 10700 \mathrm{fs}^{2}$ and a simple pulse propagation model, considering only GVD, a signal pulse duration of $\sim 263 \mathrm{fs}$ is calculated, in very close agreement with the autocorrelation measurements. Implementing dispersion control using intracavity prism compressor or optimally designed chirped mirrors could lead to transform limited pulses [11].

In conclusion, we have demonstrated a femtosecond OPO for the deep-IR pumped directly by a KLM Ti:sapphire laser by exploiting critical phase-matching configuration in the new nonlinear crystal, CSP. By angle-tuning the CSP crystal, the OPO provides continuously tunable broadband radiation across 7306$8329 \mathrm{~nm}$, with FWHM bandwidths of $\sim 150 \mathrm{~nm}$ in TEMoo spatial profile. Average output powers of up to $18 \mathrm{~mW}$ have been generated at $80.5 \mathrm{MHz}$ repetition rate, equivalent to a spectral brightness of $\sim 5.6 \times 10^{20}$ photons $\mathrm{s}^{-1} \mathrm{~mm}^{-2} \mathrm{sr}^{-1} 0.1 \% \mathrm{BW}^{-1}$. The direct exploitation of the KLM Ti:sapphire laser as pump source has enabled the generation of longest idler wavelengths from CSP, and has provided record practical powers at the longest deep-IR wavelengths from this crystal, resulting in reduced complexity, improved stability, practical powers, and high beam quality. Signal pulses have a duration of $\sim 266 \mathrm{fs}$, with up to $35 \mathrm{~mW}$ extracted across 1127.8-1149.6 nm without output coupling optimization. Our results confirm that the Ti:sapphire-pumped CSP femtosecond OPO represents a highly attractive source for low-noise deep-IR spectroscopy, frequency comb generation, and medical diagnostics, using simplified pumping based on reliable and wellestablished ultrafast laser technology.

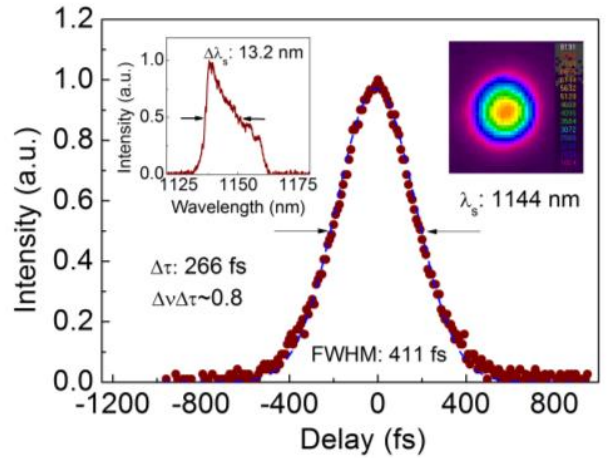

Fig. 7. Typical intensity autocorrelation of the signal pulses extracted from the CSP femtosecond OPO. Insets: Simultaneously measured signal spectrum centered at $1145 \mathrm{~nm}$, TEM 00 beam profile at 1144 $\mathrm{nm}$.

We acknowledge support from Spanish Ministry of Economy and Competitiveness (MINECO) (nuOPO, TEC2015-68234-R); European Commission (Project Mid-Tech, H2020-MSCA-ITN2014); European Office of Aerospace Research and Development (EOARD) (FA9550-14-1-0390); CERCA Programme / Generalitat de Catalunya, Severo Ochoa Programme for Centres of Excellence in R\&D (SEV-2015-0522); Fundació Privada Cellex.

Callum F. O'Donnell acknowledges the support of Marie Curie Actions: Innovative Training Network through the Mid-Tech project (H2020-MSCA-ITN-2014).

\section{References}

1. J. M. Bakker, L. Mac Aleese, G. Meijer and G. von Helden, Phys. Rev. Lett. 91, 203003 (2003).

2. A. Schliesser, N. Picqué, T. W. Hänsch, Nat. Photonics 6, 440-449 (2012).

3. C. Petibois and G. Deleris, Trends Biotechnol. 24, 455 (2006).

4. I. Coddington, W. C. Swann and N. R. Newbury, Phys. Rev. A. 82, 043817 (2010).

5. F. Adler, K. C. Cossel, M. J. Thorpe, I. Hartl, M. E. Fermann, and J. Ye, Opt. Lett. 34, 1330 (2009).

6. K. T. Zawilski, P. G. Schunemann, T. M. Pollak, D. E. Zelmon, N. C. Fernelius, and F. K. Hopkins, J. Cryst. Growth 312, 1127 (2010).

7. S. Chaitanya Kumar, P. G. Schunemann, K. T. Zawilski, and M. EbrahimZadeh, J. Opt. Soc. Am. B. 33, D44 (2016).

8. Z. Zhang, D. T. Reid, S. Chaitanya Kumar, M. Ebrahim-Zadeh, P. G. Schunemann, K. T. Zawilski and C. R: Howle, Opt. Lett. 38, 5110 (2013).

9. S. Chaitanya Kumar, J. Krauth, A. Steinmann, K. T. Zawilski, P. G. Schunemann, H. Giessen and M. Ebrahim-Zadeh, Opt. Lett. 40, 1398 (2015).

10. V. Ramaiah-Badarla, S. Chaitanya Kumar, A. Esteban-Martin, K. Devi, K. T. Zawilski, P. G. Schunemann, and M. Ebrahim-Zadeh, Opt. Lett. 41, 1708 (2016).

11. S. Chaitanya Kumar, A. Esteban-Martin, A. Santana, K. T. Zawilski, P. G. Schunemann, and M. Ebrahim-Zadeh, Opt. Lett. 41, 3355 (2016).

12. L. Maidment, P. G. Schunemann, and D. T. Reid, Opt. Lett. 41, 4261-4264 (2016).

13. P. Dumas, F. Polack, B. Lagarde, O. Chubar, J. L. Giorgetta, and S. Lefrançois, Infrared Phys. Techn. 49, 152 (2006).

14. Y. Yu, X. Gai, P. Ma, D. Choi, Z. Yang, R. Wang, S. Debbarma, S. Madden and B. Luther-Davies, Laser Photonics Rev. 8, 792 (2014). 


\section{References (With Titles)}

[1] J. M. Bakker, L. Mac Aleese, G. Meijer and G. von Helden, "Fingerprint IR spectroscopy to probe amino acid conformations in the gas phase," Phys. Rev. Lett. 91 (20), 203003 (2003).

[2] A. Schliesser, N. Picqué, T. W. Hänsch, "Mid-infrared frequency combs," Nat. Photonics 6, 440-449 (2012).

[3] C. Petibois and G. Deleris, "Chemical mapping of tumor progression by FT-IR imaging: towards molecular histopathology," Trends Biotechnol. 24, 455-462 (2006).

[4] I. Coddington, W. C. Swann and N. R. Newbury, "Coherent dualcomb spectroscopy at high signal-to-noise ratio," Phys. Rev. A. 82, 043817 (2010).

[5] F. Adler, K. C. Cossel, M. J. Thorpe, I. Hartl, M. E. Fermann, and J. Ye, "Phase-stabilized, $1.5 \mathrm{~W}$ frequency comb at 2.8-4.8 $\mu \mathrm{m}$," Opt. Lett. 34, 1330-1332 (2009).

[6] K. T. Zawilski, P. G. Schunemann, T. M. Pollak, D. E. Zelmon, N. C. Fernelius, and F. K. Hopkins, "Growth and characterization of large $\mathrm{CdSiP}_{2}$ single crystals," J. Cryst. Growth 312, 1127-1132 (2010).

[7] S. Chaitanya Kumar, P. G. Schunemann, K. T. Zawilski, and M. Ebrahim-Zadeh, "Advances in ultrafast optical parametric sources for the mid-infrared based on CdSiP2," J. Opt. Soc. Am. B. 33, D44-D56.

[8] Z. Zhang, D. T. Reid, S. Chaitanya Kumar, M. Ebrahim-Zadeh, P. G. Schunemann, K. T. Zawilski and C. R: Howle, "Femtosecond-laser pumped CdSiP2 optical parametric oscillator producing $100 \mathrm{MHz}$ pulses centered at $6.2 \mu \mathrm{m}$," Opt. Lett. 38, 5110-5113 (2013).

[9] S. Chaitanya Kumar, J. Krauth, A. Steinmann, K. T. Zawilski, P. G. Schunemann, H. Giessen and M. Ebrahim-Zadeh, "High-power femtosecond mid-infrared optical parametric oscillator at $7 \mu \mathrm{m}$ based on $\mathrm{CdSiP}_{2}$," Opt. Lett. 40, 1398-1401 (2015).

[10] V. Ramaiah-Badarla, S. Chaitanya Kumar, A. Esteban-Martin, K. Devi, K. T. Zawilski, P. G. Schunemann, and M. Ebrahim-Zadeh, "Ti:sapphire-pumped deep-infrared femtosecond optical parametric oscillator based on CdSiP,", Opt. Lett. 41, 1708- 1711 (2016).

[11] S. Chaitanya Kumar, A. Esteban-Martin, A. Santana, K. T. Zawilski, P. G. Schunemann, and M. Ebrahim-Zadeh, "Pump-tuned deepinfrared femtosecond optical parametric oscillator across 6-7 $\mu \mathrm{m}$ based on $\mathrm{CdSiP}_{2}$," Opt. Lett. 41, 3355-3358 (2016).

[12] L. Maidment, P. G. Schunemann, and D. T. Reid, "Molecular fingerprint-region spectroscopy from 5 to $12 \mu \mathrm{m}$ using an orientationpatterned gallium phosphide optical parametric oscillator," Opt. Lett. 41, 4261-4264 (2016).

[13] P. Dumas, F. Polack, B. Lagarde, O. Chubar, J. L. Giorgetta, and S. Lefrançois, "Synchrotron infrared microscopy at the French Synchrotron Facility SOLEIL," Infrared Phys. Techn. 49, 152 (2006).

[14]Y. Yu, X. Gai, P. Ma, D. Choi, Z. Yang, R. Wang, S. Debbarma, S. Madden and B. Luther-Davies, "A broadband, quasi-continuous, mid-infrared supercontinuum generated in a chalcogenide glass waveguide," Laser Photonics Rev. 8, 792-798 (2014). 